\title{
Primer registro de Dolioletta gegenbauri (Uljanin, 1884) y Doliolum nationalis Borgert, 1893 en aguas chilenas (Tunicata, Doliolida)
}

\author{
Pedro Apablaza \& Sergio Palma \\ Escuela de Ciencias del Mar, Pontificia Universidad Católica de Valparaíso \\ Casilla 1020, Valparaíso, Chile
}

RESUMEN. Se registra por primera vez en aguas chilenas la presencia de Dolioletta gegenbauri (Uljanin, 1884) y Doliolum nationalis Borgert, 1893 del orden Doliolida. Los organismos analizados corresponden a 31 ejemplares de Dolioletta gegenbauri, distribuidos en 8 forozooides, 15 gonozooides y 8 nodrizas, y 20 ejemplares de Doliolum nationalis, todos ellos forozooides. Todos los organismos se colectaron entre la superficie y los $100 \mathrm{~m}$ de profundidad, al interior de la bahía de Mejillones ( $\left.23^{\circ} \mathrm{S}\right)$, en octubre de 2001.

Palabras clave: Dolioletta gegenbauri, Doliolum nationalis, Doliolida, Mejillones, Chile.

\section{First record of Dolioletta gegenbauri (Uljanin, 1884) and Doliolum nationalis Borgert, 1893 for Chilean waters (Tunicata, Doliolida)}

\begin{abstract}
Dolioletta gegenbauri (Uljanin, 1884) and Doliolum nationalis Borgert, 1893 are reported for the first time for Chilean waters. The organisms studied included 31 specimens of Dolioletta gegenbauri, divided among 8 phorozooids, 15 gonozooids and 8 nurses, plus 20 specimens of Doliolum nationalis, which were all phorozooids. All the specimens were collected in Mejillones Bay, Chile $\left(23^{\circ} \mathrm{S}\right)$ between 0 and $100 \mathrm{~m}$ depth, in October 2001.
\end{abstract}

Key words: Dolioletta gegenbauri, Doliolum nationalis, Doliolida, Mejillones, Chile.

La clase Thaliacea, conformada por salpas, doliólidos y pyrosomas, son organismos filtradores holoplanctónicos, frecuentemente son uno de los mayores componentes de la biomasa zooplanctónica en la capa superficial más cálida y templada de los océanos (Blackburn, 1979).

Los doliólidos, son el grupo menos estudiado de la clase Thaliacea, a pesar que sus primeros registros datan de 1835, cuando Quoy y Gaimard se refirieron al género Doliolum en su reporte sobre la Expedición “Astrolabe” (1826-1829) al Pacífico tropical. El estudio de estos tunicados se intensificó en la segunda mitad del siglo XX, cuando los científicos comenzaron a comprender el complejo ciclo de vida de estos organismos (Esnal \& Daponte, 1999).
Estos organismos se caracterizan por presentar una alternancia de generación asexual u oozooide y sexual o blastozooide, como es típico de los Thaliacea, pero entre los blastozooides aparecen varias formas diferentes sucesivas (gastrozooides, forozooides, gonozooides) que complican su ciclo reproductivo (Esnal \& Daponte, 1999).

Los doliólidos, como todos los tunicados, están especializados para ingerir partículas del nanoplancton $(<20 \mathrm{~mm})$, pero a diferencia de otros tunicados, como las salpas, los mecanismos de natación y filtración están separados, lo que les permite alimentarse sin desplazarse y así disminuir su gasto energético. Por otra parte, estudios en la costa de Japón indican que algunos tunicados, como $D$. 
gegenbauri son importantes productores secundarios en la cadena de carbono de los océanos, con una producción media de $8,4 \mu \mathrm{gC} \cdot \mathrm{L}^{-1}$, el doble de la de los copépodos calanoides (Nakamura, 1998).

La mayor parte de las especies conocidas se han colectado en zonas templadas del océano Atlántico y mar Mediterráneo, siendo las más frecuentes y abundantes, Doliolum denticulatum, Doliolina muelleri y Dolioletta gegenbauri. En el Pacífico suroriental la información sobre estos organismos es casi inexistente y su mención en trabajos científicos no menciona las especies encontradas (Mujica \& Rojas, 1985; Mujica, 1993).

Las preferencias ecológicas de estos organismos los definen como buenos indicadores de masas de aguas, es así como Doliolum denticulatum está limitado por bajas temperaturas $\left(14-15^{\circ} \mathrm{C}\right)$, siendo buen indicador de aguas cálidas de la corriente de Agulhas. Un carácter similar muestra Doliolina muelleri que presenta una distribución limitada por la isoterma de $15^{\circ} \mathrm{C}$, mientras que Dolioletta gegenbauri ha sido colectado en la corriente de Benguela con un límite de distribución a los $12,8^{\circ} \mathrm{C}$; el resto de las especies son menos frecuentes y abundantes (Esnal \& Daponte, 1999).

\section{Dolioletta gegenbauri (Uljanin, 1884)}

Material examinado: se analizó un total de 31 especímenes, distribuidos en 8 forozooides, 15 gonozooides y 8 nodrizas colectados en pescas verticales entre 0-100 m con redes WP-2 de $57 \mathrm{~cm}$ de diámetro de boca y $200 \mu \mathrm{m}$ de abertura de malla, en octubre de 2001 en la bahía de Mejillones $\left(23^{\circ} \mathrm{S}\right)$. La talla corporal fluctuó en forozooides entre 3,7 y 7,0 $\mathrm{mm}$, con una media de 5,4 $\pm 0,9 \mathrm{~mm}$, en gonozooides entre 3,1 y $8,6 \mathrm{~mm}$, con una media de $5,9 \pm 4,5 \mathrm{~mm}$, y en nodrizas entre 6,6 y $8,9 \mathrm{~mm}$, con una media de 7,8 $\pm 0,9 \mathrm{~mm}$. Estas tallas son menores a las observadas en el océano Atlántico, donde las nodrizas pueden alcanzar longitudes superiores a $4 \mathrm{~cm}$ (Esnal \& Daponte, 1999).

Diagnosis: el forozooide tiene la típica forma de barril abierto en los extremos por la presencia de los sifones bucal y cloacal, los cuales están provistos de lobulaciones. Tanto los forozooides como los gonozooides presentan ocho bandas musculares continuas. La morfología del forozooide es similar a la del gonozooide, pero carece de gónadas (Fig.1a). En el gonozooide el endostilo se extiende de M2(1/3) a M4(1/2) y el ganglio nervioso se ubica en M3(1/4). El septo-branquial se extiende dorsalmente cerca de
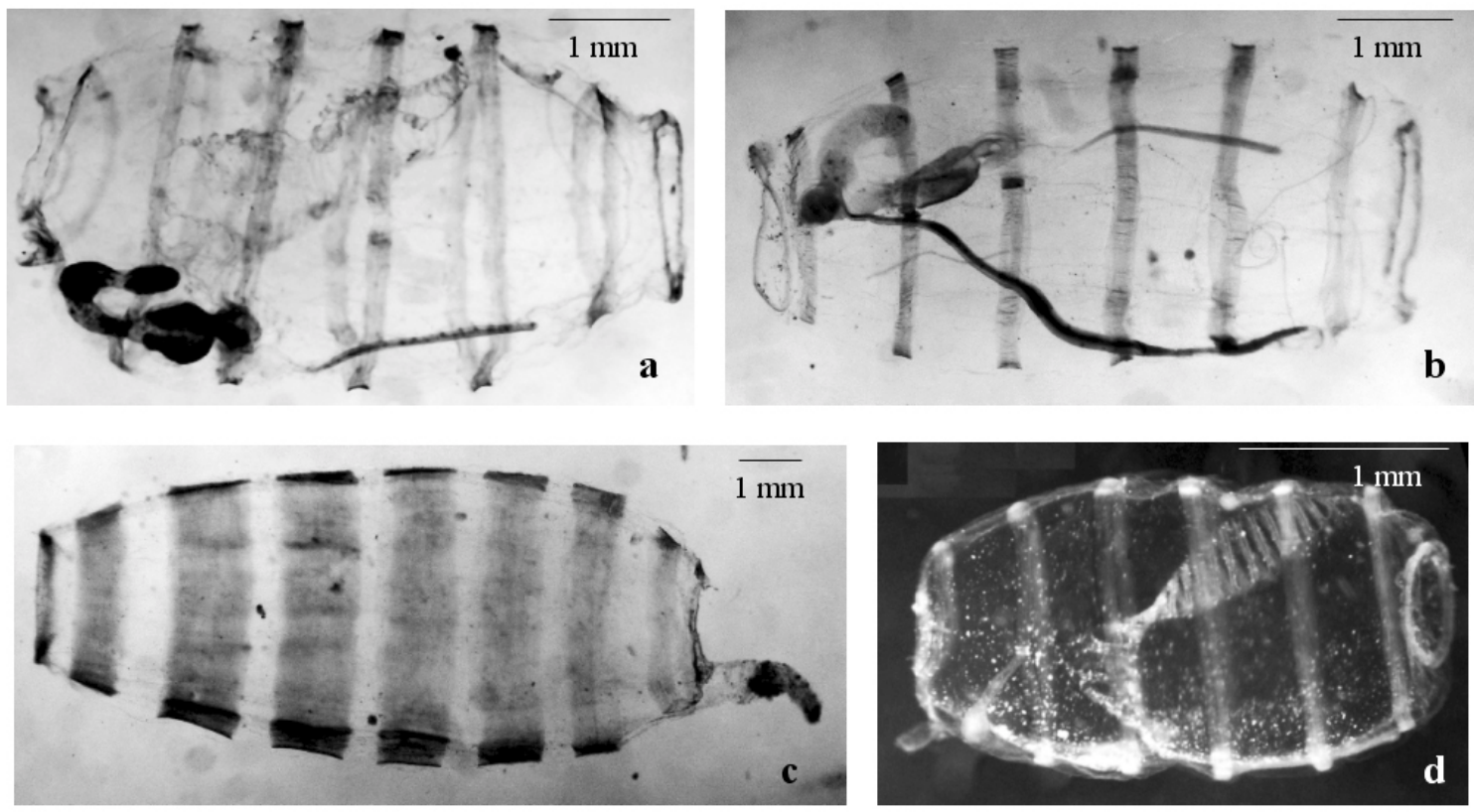

Figura 1. Ejemplares de Dolioletta gegenbauri y Doliolum nationalis. a) Dolioletta gegenbauri (forozooide), b) D. gegenbauri (gonozooide), c) D. gegenbauri (nodriza) y d) Doliolum nationalis (forozooide).

Figure 1. Specimens of Dolioletta gegenbauri and Doliolum nationalis. a) Dolioletta gegenbauri (phorozooid), b) D. gegenbauri (gonozooid), c) D. gegenbauri (nurse) and d) Doliolum nationalis (phorozooid). 
M3 y ventralmente de M5, su curvatura posterior se extiende a M5(3/4)-M6, con un gran número de hendiduras, aproximadamente 60 . El ovario es piriforme y está ubicado cerca de M7. El testículo tiene forma de estaca y se ubica próximo al ovario y se extiende dorsalmente hasta M2 o más allá (Fig. 1b). En las nodrizas las bandas musculares M3 y M4 son más anchas (Fig. 1c).

Distribución: D. gegenbauri es una de las tres especies más frecuentes y abundantes del orden Doliolida. Habita exclusivamente en aguas epipelágicas (0-200 m) y las mayores densidades se registran en los primeros $50 \mathrm{~m}$ (Berner, 1960).

Aunque en un principio se consideró como una especie oceánica cosmopolita, algunos autores mencionan cierta tendencia a incrementar su abundancia hacia la costa, lo que es corroborado por Paffenhöfer et al. (1991), quienes registraron altas densidades en aguas costeras de la península de Florida, Atlántico norte. Anteriormente, en 1957 y 1958 se reportó la presencia de $D$. gegenbauri en el estrato de 70-140 m de profundidad al sur de la península, donde se encuentra asociada a aguas costeras frías de la corriente de California (Berner, 1960). Blackburn (1979) señala que en la corriente de California, esta especie está limitada por las isotermas de $14^{\circ} \mathrm{C}$ en los meses fríos y de $16^{\circ} \mathrm{C}$ en los meses cálidos. De manera similar, en el Atlántico sur ha sido colectada en la corriente de Benguela por Neumann en 1906, asociada a la isoterma de $12,8^{\circ} \mathrm{C}$ (Esnal \& Daponte, 1999).

\section{Doliolum nationalis Borgert, 1893}

Material analizado: se identificó un total de 20 forozooides colectados en pescas verticales entre 0 $100 \mathrm{~m}$ con redes WP-2 de $57 \mathrm{~cm}$ de diámetro de boca y $200 \mu \mathrm{m}$ de abertura de malla, en la bahía de Mejillones $\left(23^{\circ} \mathrm{S}\right)$, en octubre de 2001. El tamaño de estos ejemplares fluctuó entre 1,2 y $3,3 \mathrm{~mm}$, con una media de $2,3 \pm 0,5 \mathrm{~mm}$.

Diagnosis: al igual que Dolioletta gegenbauri el forozooide tiene la forma típica de barril abierto en los extremos, los cuales están provistos de lobulaciones. Tanto los forozooides como los gonozooides presentan ocho bandas musculares continuas. La morfología del forozooide es similar a la del gonozooide, pero sin gónadas. El forozooide presenta un endostilo corto que se extiende desde M2 a M4 y el ganglio nervioso se ubica en M3(3/4).
El septo branquial está fuertemente arqueado, se extiende dorsalmente a M2 y ventralmente a M5, su curvatura posterior se extiende hasta M5(1/2). El tubo digestivo forma un arco dextral ancho alrededor de la base de la cloaca (Fig. 1d).

Distribución: habita preferentemente en aguas neríticas y ha sido registrada en aguas ecuatoriales y tropicales del océano Atlántico y mar Mediterráneo y su abundancia parece declinar hacia aguas más frías (Esnal \& Daponte, 1999). Esta especie es capaz de subsistir bajo severas condiciones de temperatura y salinidad, y su distribución vertical está limitada a los primeros $50 \mathrm{~m}$ de profundidad (Godeaux, 1977, 1987).

En ciertas regiones del Mediterráneo, $D$. nationalis, presenta particularidades como la presencia de solo la fase forozooide. La causa es desconocida, aunque algunas hipótesis mencionan razones tróficas o la ausencia de alguna vitamina, mientras que en otras regiones, como en el golfo de Lion y Villefranche-sur-Mer, se han observado gonoforozooides, que son el resultado del forozooide normalmente estéril, que desarrolla gónadas (Godeaux, 1988).

La ausencia de gonozooides es un rasgo interesante en esta especie, que ha sido mencionado por varios autores. Al respecto, Braconnot (1967) sugirió que esta especie tiene un ciclo de vida corto en el Mediterráneo, donde la etapa gonozooide está ausente. Posteriormente, Esnal et al. (1982) infirieron esto mismo para la costa occidental de Florida, donde también encontraron una desproporción en la relación entre forozooides y gonozooides, siendo los primeros mucho más frecuentes y abundantes. En consecuencia, es probable que la ausencia de gonozooides en la bahía de Mejillones pueda también ser indicativa de un ciclo de vida corto, al igual que en el Mediterráneo y costa occidental de Florida.

En la bahía de Mejillones, los especímenes pertenecientes a Dolioletta gegenbauri y D. nationalis estuvieron asociados a aguas subtropicales con altos valores de temperatura $\left(16-17^{\circ} \mathrm{C}\right)$, salinidad (34,0-34,5 psu) y contenido de oxígeno disuelto (5 $\left.\mathrm{mL} \cdot \mathrm{L}^{-1}\right)$. Finalmente, se puede concluir que la presencia de Dolioletta gegenbauri y Doliolum nationalis en la bahía de Mejillones, constituye el primer registro del orden Doliolida en aguas chilenas y contribuye al conocimiento de la biodiversidad zooplanctónica del Pacífico suroriental. 


\section{AGRADECIMIENTOS}

Los autores agradecen el financiamiento otorgado por CONICYT a través del Proyecto FONDECYT 1000419, que permitió el desarrollo de esta investigación.

\section{REFERENCIAS}

Berner, L.D. 1960. Unusual features in the distribution of pelagic tunicates in 1957 and 1958. CalCOFI Rep., 7: 133-135.

Blackburn, M. 1979. Thaliacea of the California current region: relations to temperature, chlorophyll, currents and upwelling. CalCOFI Rep., 20: 184-214.

Braconnot, J.C. 1967. Sur la possibilité d'un cycle court de développment chez le tunicier pélagique: Doliolum nationalis Borget, 1893. C.R. Acad. Sci. Paris, 264: 1434-1437.

Esnal, G. \& M. Daponte. 1999. Doliolida. En: D. Boltovskoy (ed). South Atlantic Zooplankton. Backhuys Publishers, Leiden. Vol. 2, pp 1.4091.421 .

Esnal, G., R.J. Castro \& L.C. Simone. 1982. Sobre sálpidos y doliólidos (Tunicata, Tahaliacea) de las costas de Florida, con un aporte a la diferencia- ción morfométrica de las nodrizas de Dolioletta y Doliolina. Physis, Secc. A, 40(99): 63-68.

Godeaux, J. 1977. Thaliacea from off the coasts of Tropical West Africa. Atlantide Rep., 12: 7-24.

Godeaux, J. 1987. Distribution of Thaliacea on a transect from the Gulf of Aden to the Central Red Sea during the winter monsoon (March 1979). Oceanol. Acta, 10(2): 197-204.

Godeaux, J. 1988. Thaliacea Méditerranéens: une synthèse. Bull. Soc. Roy. Sci. Liège, 57(4-5): 359377.

Mujica, A. 1993. Zooplancton de las aguas circundantes a isla de Pascua $\left(27^{\circ} 08^{\prime} \mathrm{S}-109^{\circ} 26^{\prime} \mathrm{W}\right)$. Cienc. Tecnol. Mar, 16: 55-61.

Mujica, A. \& O. Rojas. 1985. Composición y abundancia del zooplancton del norte de Chile. Crucero Mar Chile XV-ERFEN VI, Diciembre 1982. Cienc. Tecnol. Mar, 9: 31-52.

Nakamura Y. 1998. Blooms of tunicates Oikopleura spp. and Dolioletta gegenbauri in the Seto Inland Sea, Japan, during summer. Hydrobiologie, 385(13): 183-192.

Paffen höfer G.A., T.B. Stewart, M.J. Youngbluth \& T.G. Bailey. 1991. High-resolution vertical profiles of pelagic tunicates. J. Plankton Res., 13: 971-981. 